\title{
LINEAR OPERATORS THAT PRESERVE COLUMN RANK OF BOOLEAN MATRICES
}

\author{
SEOK-ZUN SONG
}

(Communicated by Eric Friedlander)

\begin{abstract}
We study the extent to which certain theorems on rank preservers of Boolean matrices carry over to column rank preservers. We characterize the linear operators that preserve the column rank of Boolean matrices.
\end{abstract}

\section{INTRODUCTION AND PRELIMINARIES}

There are many papers on the study of linear operators that preserve the rank of matrices over several semirings. Boolean matrices also have been the subject of research by many authors. But there are few papers on column rank preservers of the matrices over semirings. Recently Beasley and Song [1] obtained characterizations of column rank preservers of matrices over nonnegative integers.

In this paper, we obtain characterizations of the linear operators that preserve the column rank of Boolean matrices.

We let $\mathbb{M}_{m, n}(B)$ denote the set of all $m \times n$ matrices with entries in $B=$ $\{0,1\}$, the two element Boolean algebra. Arithmetic in $B$ follows the usual rules except that $1+1=1$. Throughout this paper, we shall adopt the convention that $m \leq n$.

If $\mathbb{V}$ is a nonempty subset of $B^{k}=\mathbb{M}_{k, 1}(B)$ containing 0 which is closed under addition, then $\mathbb{V}$ is called a Boolean vector space. If $\mathbb{V}$ and $\mathbb{W}$ are vector spaces with $\mathbb{V} \subset \mathbb{W}$, then $\mathbb{V}$ is called a subspace of $\mathbb{W}$. We identify $\mathbb{M}_{m, n}(B)$ with $B^{m n}$ in the usual way when we discuss it as a Boolean vector space and consider its subspaces.

Let $\mathbb{V}$ be a Boolean vector space. If $S$ is a subset of $\mathbb{V}$, then $\langle S\rangle$ denotes the intersection of all subspaces of $\mathbb{V}$ containing $S$, which is a subspace of $\mathbb{V}$ too, called the subspace generated by $S$. If $S=\left\{V_{1}, V_{2}, \ldots, V_{r}\right\}$, then $\langle S\rangle=\left\{\sum_{i=1}^{r} x_{i} v_{i} \mid x_{i} \in B\right\}$, the set of linear combinations of elements in $S$.

Received by the editors April 8, 1992; presented at the conference of the International Linear Algebra Society at the University of West Florida, March 17-20, 1993.

1991 Mathematics Subject Classification. Primary 15A03, 15 A04.

Key words and phrases. Column rank, congruence operator.

This paper was supported by the NON-DIRECTED RESEARCH FUND, Korea Research Foundation, 1991, and in part by KOSEF-TGRC. 
Note that $\langle\varnothing\rangle=\{0\}$. Define the dimension of $\mathbb{V}$, written $\operatorname{dim}(\mathbb{V})$, to be the minimum of the cardinalities of all subsets $S$ of $\mathbb{V}$ generating $\mathbb{V}$. We call a generating set of cardinality equal to $\operatorname{dim}(\mathbb{V})$ a basis of $\mathbb{V}$. It is a known fact that every Boolean vector space has only one basis.

A subset of $\mathbb{V}$ is called linearly independent if none of its members is a linear combination of the others. Evidently every basis is independent.

The subspaces of $B^{m}$ generated by the columns of an $m \times n$ Boolean matrix $A$ is called the column space of $A$. We define the column rank of $A, c(A)$, to be the dimension of the column space of $A$. If an $m \times n$ Boolean matrix $A$ is not zero, then its Boolean rank, $b(A)$, is the least $k$ for which there exist $m \times k$ and $k \times n$ Boolean matrices $F$ and $G$ with $A=F G$. The Boolean rank of the zero matrix is 0 .

It follows that $0 \leq b(A) \leq c(A) \leq n$ for arbitrary $A \in \mathbb{M}_{m, n}(B)$.

Beasley and Pullman [2] showed the following:

Lemma 1.1 [2]. If the columns of $A \in \mathbb{M}_{m, n}(B)$ are linearly independent, then $c(A)=n$.

Lemma 1.2 [2]. Let $\mu(B, m, n)$ be the largest integer $k$ such that, for all $m \times n$ Boolean matrices, $r(A)=c(A)$ if $r(A) \leq k$. Then

$$
\mu(B, m, n)= \begin{cases}1 & \text { if } \min (m, n)=1, \\ 3 & \text { if } m \geq 3 \text { and } n=3, \\ 2 & \text { otherwise. }\end{cases}
$$

\section{COLUMN RANK PRESERVERS OF BoOleAN MATRICES}

If $\mathbb{V}$ is a Boolean vector space, a mapping $T: \mathbb{V} \rightarrow \mathbb{V}$ which preserves sums and 0 is said to be a (Boolean) linear operator.

Suppose $T$ is a linear operator on $\mathbb{M}_{m, n}(B)$. Say that $T$ is a:

(i) congruence operator if there exist invertible matrices $U$ and $V$ in $\mathbb{M}_{m, n}(B)$ such that $T(A)=U A V$ for all $A$ in $\mathbb{M}_{m, n}(B)$;

(ii) transposition operator if $m=n$ and $T(A)=A^{t}$, the transpose matrix of $A$, for all $A$ in $\mathbb{M}_{m, m}(B)$;

(iii) column rank preserver if $c(T(A))=c(A)$ for all $A$ in $\mathbb{M}_{m, n}(B)$.

Also, $T$ preserves column rank $k$ if $c(T(A))=k$ whenever $c(A)=k$ for all $A$ in $\mathbb{M}_{m, n}(B)$.

Rank preservers are defined in a manner similar to (iii). We define a subspace of $\mathbb{M}_{m, n}(B)$ whose nonzero members have Boolean column rank 1 as a columnrank-1 space.

Using Lemma 1.2, we can apply the results for ranks 1 and 2 in [3] to those for column ranks 1 and 2. Thus we obtain the following Theorem 2.1 and Lemma 2.1 by proofs analogous to those in [3].

Theorem 2.1. If $T$ is a linear operator on $\mathbb{M}_{m, n}(B)$, then the following statements are equivalent:

(a) $T$ is injective and preserves column rank 1 .

(b) $T$ preserves the column ranks 1 and 2 and preserves the dimension of all column-rank-1 spaces. 
(c) $T$ is in the group of operators generated by congruence and transposition operators.

Lemma 2.1. If $T$ is a linear operator on $\mathbb{M}_{m, n}(B)$ with $m>1$ and $T$ is not injective but preserves column rank 1 , then $T$ decreases the column rank of some column rank 2 matrix.

But some rank preservers do not preserve any column ranks as shown in Lemma 2.2 below.

Example 2.1. Consider

$$
A=\left(\begin{array}{llll}
1 & 1 & 0 & 1 \\
1 & 0 & 1 & 0 \\
0 & 1 & 1 & 0 \\
1 & 1 & 1 & 1
\end{array}\right) .
$$

Then none of the columns of $A$ are linear combinations of the others. So $c(A)=4$ by Lemma 1.1. But $c\left(A^{t}\right)=3$ because the fourth column of $A^{t}$ is the sum of the first and second columns of $A^{t}$, and $b(A)=b\left(A^{t}\right) \leq c\left(A^{t}\right)=3$. By Lemma $1.2 b(A)$ is greater than 2. So $b(A)=3$. This shows that, for $A \in \mathbb{M}_{m, m}(B)$ with $m \geq 4, b(A)<c(A)$ is possible.

Lemma 2.2. If $T$ is a transposition operator on $\mathbb{M}_{m, m}(B)$ with $m \geq 4$, then $T$ does not preserve column rank $c$ for $c \geq 3$ but preserves all Boolean ranks.

Proof. Let $A$ be the matrix in Example 2.1. Consider $C=A \oplus 0_{m-4} \in$ $\mathbb{M}_{m, m}(B)$. Then $c\left(C^{t}\right)=3$ but $T\left(C^{t}\right)=C$ has column rank 4 . Let

$$
D=A \oplus I_{k} \oplus 0_{m-k-4} \in \mathbb{M}_{m, m}(B),
$$

where $I_{k}$ is the identity matrix of order $k$. Then $c\left(D^{t}\right)=3+k$ but $T\left(D^{t}\right)=D$ has column rank $4+k$. Therefore, $T$ does not preserve column rank $c$ for $c \geq 3$, but it is obvious that $T$ preserves all Boolean ranks.

Theorem 2.2. Suppose $T$ is a linear operator on $\mathbb{M}_{m, n}(B)$ for $n \geq m \geq 4$. Then the following statements are equivalent:

(a) $T$ preserves column ranks 1,2 , and 3.

(b) $T$ is a congruence operator.

(c) $T$ is a column rank preserver.

(d) $T$ is bijective and preserves column ranks 1 and 3 .

Proof. (a) implies (b): Since $T$ preserves column ranks 1 and 2, $T$ is injective by the contraposition of Lemma 2.1. Theorem 2.1 implies that $T$ is in the group of operators generated by congruence and transposition operators. Since a transposition operator does not preserve column rank 3 by Lemma 2.2, $T$ is a congruence operator.

(b) implies (c): If $T$ is a congruence operator, then there exist invertible matrices $U$ and $V$ of proper orders over $B$ such that $T(A)=U A V$ for $A \in \mathbb{M}_{m, n}(B)$. Since the invertible matrices $U$ and $V$ are permutation matrices over $B, U A$ and $A V$ do not change column rank of $A$. So $T$ preserves all column ranks of matrices in $\mathbb{M}_{m, n}(B)$.

It is obvious that (c) implies (a); Theorem 2.1 and Lemma 2.2 imply that (b) and (d) are equivalent. 
Thus we have characterizations of the linear operators that preserve column rank of Boolean matrices when $n \geq m \geq 4$. They are different from the characterizations of the linear operator that preserves Boolean rank in [3]. For $n \leq 3$, they are the same as the characterizations of the rank preservers by Lemma 1.2.

\section{REFERENCES}

1. L. B. Beasley and S. Z. Song, A comparison of nonnegative real ranks and their preservers, Linear and Multilinear Algebra 31 (1992), 37-46.

2. L. B. Beasley and N. J. Pullman, Semiring rank versus column rank, Linear Algebra Appl. 101 (1988), 33-48.

3. __ Boolean-rank-preserving operators and Boolean-rank-1 spaces, Linear Algebra Appl. 59 (1984), 55-77.

Department of Mathematics, Cheju National University, Cheju, 690-756, Republic of KOREA 